\title{
Patterns in blood pressure medication use in US incident dialysis patients over the first 6 months
}

\author{
Wendy L St. Peter ${ }^{1,2^{*}}$, Stephen M Sozio ${ }^{3,4}$, Tariq Shafi ${ }^{3,4}$, Patti L Ephraim ${ }^{4,5}$, Jason Luly ${ }^{6}$, Aidan McDermott ${ }^{7}$, \\ Karen Bandeen-Roche ${ }^{7}$, Klemens B Meyer ${ }^{8}$, Deidra C Crews ${ }^{3,4}$, Julia J Scialla ${ }^{9}$, Dana C Miskulin ${ }^{8}$, Navdeep Tangri ${ }^{10}$, \\ Bernard G Jaar ${ }^{3,4,5,11}$, Wieneke M Michels ${ }^{4,6,12}$, Albert W Wu $u^{5,13,14,15}$, and L Ebony Boulware ${ }^{4,5,6}$ for the DEcIDE \\ Network Patient Outcomes in End-Stage Renal Disease Study Investigators
}

\begin{abstract}
Background: Several observational studies have evaluated the effect of a single exposure window with blood pressure (BP) medications on outcomes in incident dialysis patients, but whether BP medication prescription patterns remain stable or a single exposure window design is adequate to evaluate effect on outcomes is unclear.

Methods: We described patterns of BP medication prescription over 6 months after dialysis initiation in hemodialysis and peritoneal dialysis patients, stratified by cardiovascular comorbidity, diabetes, and other patient characteristics. The cohort included 13,072 adult patients (12,159 hemodialysis, 913 peritoneal dialysis) who initiated dialysis in Dialysis Clinic, Inc., facilities January 1, 2003-June 30, 2008, and remained on the original modality for at least 6 months. We evaluated monthly patterns in BP medication prescription over 6 months and at 12 and 24 months after initiation.

Results: Prescription patterns varied by dialysis modality over the first 6 months; substantial proportions of patients with prescriptions for beta-blockers, renin angiotensin system agents, and dihydropyridine calcium channel blockers in month 6 no longer had prescriptions for these medications by month 24. Prescription of specific medication classes varied by comorbidity, race/ethnicity, and age, but little by sex. The mean number of medications was 2.5 at month 6 in hemodialysis and peritoneal dialysis cohorts.
\end{abstract}

Conclusions: This study evaluates BP medication patterns in both hemodialysis and peritoneal dialysis patients over the first 6 months of dialysis. Our findings highlight the challenges of assessing comparative effectiveness of a single BP medication class in dialysis patients. Longitudinal designs should be used to account for changes in BP medication management over time, and designs that incorporate common combinations should be considered.

Keywords: Blood pressure medication, Dialysis, Medication use patterns

\section{Background}

The mortality rate for incident dialysis patients is high in the first few months. A large proportion of these deaths are due to cardiovascular causes, which remain the leading causes of death after the first 6 months [1]. Hypertension, congestive heart failure (CHF), and atherosclerotic heart disease occur in $85 \%, 32 \%$, and $21 \%$, respectively, of incident dialysis patients [1]. Two

\footnotetext{
* Correspondence: wstpeter@cdrg.org

'University of Minnesota College of Pharmacy, Minneapolis, MN, USA

${ }^{2}$ Chronic Disease Research Group, Minneapolis Medical Research Foundation, 914 South 8th Street, Suite S4.100, Minneapolis, MN 55404, USA

Full list of author information is available at the end of the article
}

meta-analyses of small randomized clinical trials in dialysis patients receiving blood pressure (BP) medications suggest that BP treatment decreased cardiovascular events compared with control or placebo groups [2,3], particularly among patients with hypertension [2]. However, heterogeneity among the trials was substantial, and these analyses could not determine differential effects among medications. Data from observational studies suggest that exposure to specific medications (calcium channel blockers, renin-angiotensin system [RAS] agents, beta-blockers) is associated with reduced allcause or cardiovascular mortality compared with no BP medication use [4-11]. However, clinical trials have been

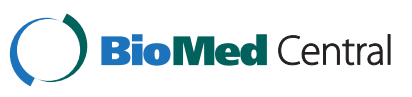


limited in their ability to conclusively support use of specific agents because of small sample sizes and heterogeneity of study designs. Observational studies to date have also been limited; some were conducted with prevalent patients with varying dialysis duration and comorbid conditions, possibly leading to problems with selection bias and confounding by indication. These concerns can be mitigated somewhat using incident populations. A more concerning issue relates to evaluation of associations based on exposure at one point in time. Understanding utilization patterns of $\mathrm{BP}$ medications in the months after dialysis initiation is imperative before appropriate designs for future studies can be determined.

However, there is a paucity of data regarding patterns of BP medication use in incident hemodialysis and peritoneal dialysis patients. Despite extensive use, no data examine longitudinal prescription patterns in the first few months of dialysis. To date, data are limited to cross-sectional analyses of prevalent $[1,7,11-15]$ or incident dialysis patients $[8,9,16]$. In addition, these cross-sectional analyses have either focused on hemodialysis patients or have grouped all dialysis patients together, so very little information is available specifically regarding peritoneal dialysis patients. Of the studies in incident patients, two use data from 19961997 and do not reflect current practice patterns [8,9], and one was a single small hospital study and is not generalizable [16]. Changes in BP management over the first few months of dialysis, when residual kidney function may be present and volume status is changing, is also a key consideration.

Thus, a thorough understanding of BP medication prescription patterns among incident hemodialysis and peritoneal dialysis patients, accounting for common cardiovascular conditions that could influence prescription patterns after dialysis initiation, is critical to inform future comparative-effectiveness studies. We studied prescription patterns of single and combination BP medications over the first 6 months of dialysis in hemodialysis and peritoneal dialysis patients. We hypothesized that the average number of BP medications per patient would decrease, prescriptions by medication class would change over the first 6 months, and prescription patterns would vary by dialysis modality, sex, age, race, and cardiovascular disease (CVD) or risk factors.

\section{Methods}

\section{Overview}

Our study was part of the broader Developing Evidence to Inform Decisions about Effectiveness (DEcIDE) Network Patient Outcomes in End-Stage Renal Disease (DEcIDE-ESRD) Study funded by the Agency for Healthcare Research and Quality to examine the comparative effectiveness of common treatment strategies in ESRD [17]. Cohorts were selected from patients treated in Dialysis
Clinic, Inc., (DCI) facilities 2003-2008. DCI is a not-forprofit medium-sized dialysis provider in the US with over 210 clinics in 27 states. DCI patient characteristics are similar to those of US dialysis patients in general, with an overrepresentation of black patients [1]. We linked DCI data to United States Renal Data System (USRDS) registry data to obtain additional information.

We constructed hemodialysis and peritoneal dialysis cohorts from all incident patients aged $\geq 18$ years who initiated treatment January 1, 2003-June 30, 2008, in a DCI facility and were alive at 6 months, to examine BP medication patterns in the 6 months after dialysis initiation. We excluded patients whose DCI start date occurred more than 30 days after their USRDS start date (obtained from the Medical Evidence Report, Centers for Medicare \& Medicaid [CMS] form CMS-2728), switched modalities (hemodialysis to peritoneal dialysis or vice versa, home hemodialysis, kidney transplant), transferred to a non-DCI facility, withdrew from dialysis, or were lost to follow-up during the first 6 months.

We determined presence of CVD, CHF, and diabetes at dialysis initiation from information on form CMS2728 and over the first 6 months from Medicare claims in USRDS data using International Classification of Diseases Tenth Revision codes [17]. We determined oral BP medication prescriptions from medication lists in the DCI electronic medical record (EMR). This informational system maintains a history of all medications prescribed to each DCI patient, allows physicians to write prescriptions, and generates complete patient medication lists on care plans, physician encounter forms, and transfer summaries when patients are hospitalized or receive other outpatient care. The DCI EMR does not interface with inpatient or other (non-dialysis) outpatient clinic EMRs. Each month, patients bring in their medication bottles and nurses verify, reconcile, and record the medications to update the DCI EMR. BP medications are prescribed based on individual physician discretion.

We assessed medication use in each month based on drug start and stop dates in the EMR. We used the generic product identifier code to identify and classify each medication into specific antihypertensive classes using the Wolter's Kluwer Medi-span ${ }^{\oplus}$ drug products database. We quantified the percentage of patients surviving to 6 months who were prescribed beta-blockers, RAS agents or dihydropyridine calcium channel blockers (DHP-CCB), and further quantified the percentage of surviving patients who continued to receive these classes of medications at months 12 and 24 . We also assessed BP medication prescriptions in the most recent timeframe available (2007-2008) to determine the most current trends. Intravenous BP medications administered during dialysis sessions were not included. 


\section{Statistical analyses}

We described the cohorts, numbers of BP medications, and percentages of patients with prescriptions. We used descriptive statistics (mean \pm standard deviation [SD]) for continuous variables (age, laboratory values) and percentages for categorical variables. We stratified the hemodialysis and peritoneal dialysis cohorts by modality, age, race/ethnicity, sex, CHF, CVD (atherosclerotic heart disease, cerebrovascular accident/transient ischemic attack, or peripheral vascular disease), and diabetes, as these variables have been shown to affect BP medication use [14]. We performed analyses using SAS version 9.2. We estimated all percentages to a precision with $95 \%$ confidence intervals of width $\pm 1.0 \%$ or less for hemodialysis and \pm $3.3 \%$ or less for peritoneal dialysis patients.
The Johns Hopkins Medicine Institutional Review Board (Baltimore, Maryland) reviewed and approved the study.

\section{Results}

After applying inclusion and exclusion criteria, our study cohorts consisted of 12,159 (93\%) hemodialysis and 913 (7\%) peritoneal dialysis patients, representing $62 \%$ of the initial cohort (Figure 1). Peritoneal dialysis patients were younger, less likely to be black, less likely to have ESRD caused by diabetes or hypertension, less likely to have cardiovascular conditions or diabetes, and more likely to have ESRD caused by glomerulonephritis; they had lower systolic BP and higher albumin and hemoglobin (Table 1).

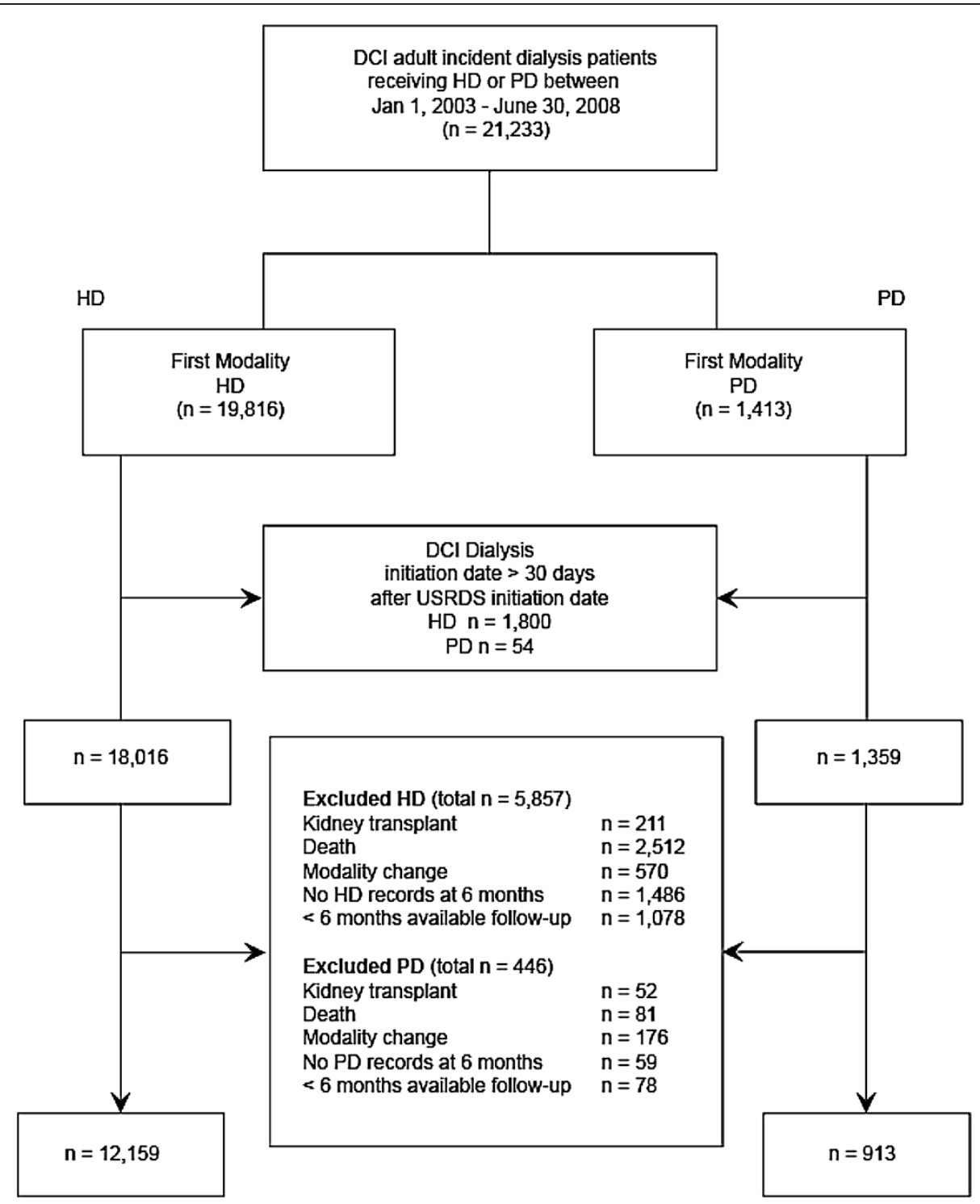

Figure 1 Flow diagram for patients included and excluded from the study. DCl, Dialysis Clinic, Inc; HD, hemodialysis; PD, peritoneal dialysis; USRDS, United States Renal Data System; patients were evaluated for exclusion criteria in the 6-month period after dialysis initiation. 


\section{Mean number of blood pressure medications and prescription patterns, first 6 months}

Most patients with any recorded medications in the EMR were prescribed BP medications by 6 months after dialysis initiation (Table 2 ). The mean $( \pm \mathrm{SD})$ number of prescribed medications was $2.8 \pm 1.4$ for peritoneal dialysis and $2.3 \pm 1.4$ for hemodialysis patients in month 1 , but by month 6 the mean was 2.5 for both cohorts; thus the mean over the first 6 months of dialysis increased for hemodialysis and decreased for peritoneal dialysis patients.

Table 1 Patient characteristics at baseline

\begin{tabular}{|c|c|c|c|}
\hline & All & Hemodialysis & $\begin{array}{c}\text { Peritoneal } \\
\text { dialysis }\end{array}$ \\
\hline$n$ & 13,072 & 12,159 & 913 \\
\hline Age, years & $61.6 \pm 15$ & $62.0 \pm 14.9$ & $56.0 \pm 14.5$ \\
\hline \multicolumn{4}{|l|}{ Sex } \\
\hline Male & 54.5 & 54.6 & 53.3 \\
\hline Female & 45.5 & 45.5 & 46.7 \\
\hline \multicolumn{4}{|l|}{ Race } \\
\hline White & 58.8 & 58.2 & 66.5 \\
\hline Black & 36.1 & 37.4 & 28.0 \\
\hline Other & 4.5 & 4.4 & 5.5 \\
\hline Hispanic & 5.4 & 5.6 & 3.6 \\
\hline \multicolumn{4}{|l|}{ ESRD cause } \\
\hline Diabetes & 48.1 & 48.5 & 43.4 \\
\hline Hypertension & 27.3 & 27.7 & 21.7 \\
\hline Glomerulonephritis & 9.5 & 9.0 & 16.9 \\
\hline Other & 15.0 & 14.8 & 18.1 \\
\hline \multicolumn{4}{|l|}{$\begin{array}{l}\text { Cardiovascular risk factors or } \\
\text { disease }\end{array}$} \\
\hline $\mathrm{CHF}$ & 43.0 & 44.7 & 20.8 \\
\hline CVD & 53.3 & 54.8 & 34.3 \\
\hline Diabetes & 62.5 & 63.3 & 51.7 \\
\hline \multicolumn{4}{|l|}{ Other characteristics } \\
\hline Systolic blood pressure & $\begin{array}{c}147.7 \pm \\
20.2\end{array}$ & $147.9 \pm 20.2$ & $144.3 \pm 19.5$ \\
\hline eGFR & $9.8 \pm 4.4$ & $9.8 \pm 4.4$ & $9.8 \pm 4.1$ \\
\hline Body mass index & $\begin{array}{l}28.7 \pm \\
7.6\end{array}$ & $28.8 \pm 7.7$ & $28.4 \pm 6.4$ \\
\hline Albumin (g/dL) & $3.1 \pm 0.7$ & $3.1 \pm 0.7$ & $3.6 \pm 0.6$ \\
\hline Hemoglobin (g/dL) & $10 \pm 1.7$ & $10.0 \pm 1.7$ & $10.8 \pm 1.5$ \\
\hline Calcium $x$ phosphorus & $\begin{array}{c}49.9 \pm \\
13.0\end{array}$ & $50.1 \pm 13.0$ & $47.8 \pm 13.3$ \\
\hline KTN & $1.5 \pm 0.3$ & $1.5 \pm 0.3$ & $1.6 \pm 0.4$ \\
\hline
\end{tabular}

Note: Values are mean \pm standard deviation or percentages. Age, eGFR, albumin, body mass index, and hemoglobin were assessed at dialysis initiation; blood pressure was the average value for each patient over month 1; calcium $x$ phosphorus and KT/V were the average value for each patient over months 4-6; cardiovascular risk factors or disease were assessed over the first 6 months.

CHF, congestive heart failure; CVD, cardiovascular disease; eGFR, estimated glomerular filtration rate; ESRD, end-stage renal disease.
The percentage of peritoneal dialysis patients with no prescribed BP medications increased from month 1 to month 6 , and the percentage of hemodialysis patients decreased slightly (Figure 2). Prescriptions for RAS agents increased in both groups. Prescriptions for DHP-CCBs remained stable for hemodialysis patients but declined for peritoneal dialysis patients. Diuretics were prescribed for $48 \%$ of peritoneal dialysis and $29 \%$ of hemodialysis patients at month 1. Diuretic prescription declined in both groups over the study timeframe. Central alpha 2 agonists (predominantly clonidine) were prescribed for 19\% of hemodialysis and peritoneal dialysis patients in month 1 ; use of these agents declined for peritoneal dialysis patients by month 6 . Minoxidil use was low in both groups. At month 6, the most highly prescribed classes were any beta-blocker (alpha-beta blockers such as carvedilol made up about one-third), any RAS agent (angiotensin-converting enzyme inhibitors [ACEIs] were twice as common as angiotensin receptor blockers [ARBs]), DHP-CCBs, diuretics, and central alpha 2 agonists.

\section{Patterns by demographic group}

Black patients were prescribed a higher mean $( \pm \mathrm{SD})$ number of BP medications than white or other-race patients $(2.7 \pm 1.4,2.3 \pm 1.3,2.4 \pm 1.3$, respectively) at month 6 . Prescriptions of any beta-blocker and of alpha-beta blockers were similar for black and white patients, but less for Hispanics. Prescription of RAS agents was similar for black and white peritoneal dialysis patients, but more frequent (particularly ACEIs) for black and Hispanic than for white hemodialysis patients. DHP-CCBs were less frequently prescribed for white hemodialysis and peritoneal dialysis patients than for other racial/ethnic groups, and loop diuretics for black patients. Alpha blocker prescriptions were similar for black and white patients, and central alpha 2 agonists, hydralazine, and minoxidil were more frequently prescribed for black than for white patients across modalities. Prescription information for other race and Hispanic ethnicity is provided for completeness, but comparisons between modalities may not be accurate for some medication classes due to few peritoneal dialysis patients in these groups (Table 2).

In general, older patients were prescribed fewer BP medications than younger patients; means at 6 months were 2.7 \pm 1.4 for ages $18-44$ years, $2.4 \pm 1.3$ for ages $65-74$ years, and $2.2 \pm 1.3$ for ages $\geq 75$ years. For hemodialysis patients, beta-blocker prescription was similar across age groups; RAS agent, DHP-CCB, central alpha 2 agonist, and minoxidil prescription declined monotonically as age increased, and alpha-blocker prescription increased monotonically. For peritoneal dialysis patients, beta-blocker prescription increased monotonically as age increased ( $46 \%$ for ages $18-44$ years, $61 \%$ for ages $\geq 75$ years), and RAS agent and DHP-CCB prescription varied across age groups. 
Table 2 Percentages of incident hemodialysis and peritoneal dialysis patients with prescriptions for specific blood pressure medications at 6 months, by race/ethnicity, 2003-2008

\begin{tabular}{|c|c|c|c|c|c|c|c|c|c|c|}
\hline \multirow[b]{2}{*}{ Medication } & \multicolumn{5}{|c|}{ Hemodialysis, $n=12,159$} & \multicolumn{5}{|c|}{ Peritoneal dialysis, $n=913$} \\
\hline & All & Black & White & Other & Hispanic & All & Black & White & Other & Hispanic \\
\hline$n$ & & 4543 & 7075 & 541 & 679 & & 256 & 607 & 50 & 33 \\
\hline None & 12 & 10 & 13 & 13 & 11 & 10 & 8 & 10 & $x x$ & $x x$ \\
\hline \multicolumn{11}{|l|}{ Beta-blocker } \\
\hline Any & 59 & 60 & 59 & 58 & 55 & 55 & 54 & 55 & 56 & 42 \\
\hline Alpha-beta & 19 & 20 & 18 & 16 & 14 & 13 & 14 & 13 & $x x$ & $x x$ \\
\hline Beta not alpha & 41 & 40 & 41 & 42 & 41 & 42 & 40 & 42 & 44 & 30 \\
\hline \multicolumn{11}{|l|}{$\operatorname{RAS}^{*}$} \\
\hline Any & 45 & 49 & 43 & 48 & 51 & 52 & 52 & 53 & 38 & 48 \\
\hline ACEI & 32 & 36 & 29 & 33 & 39 & 33 & 32 & 34 & 20 & $x x$ \\
\hline ARB & 17 & 18 & 17 & 18 & 16 & 23 & 23 & 22 & 26 & $x x$ \\
\hline \multicolumn{11}{|l|}{ CCB } \\
\hline Any & 49 & 57 & 43 & 49 & 55 & 50 & 55 & 37 & 50 & 45 \\
\hline DHP & 40 & 48 & 34 & 43 & 48 & 40 & 48 & 35 & 48 & 39 \\
\hline Non-DHP & 9 & 8 & 9 & 6 & 7 & 10 & 7 & 12 & $x x$ & $x x$ \\
\hline \multicolumn{11}{|l|}{ Diuretic $^{\dagger}$} \\
\hline Any & 28 & 23 & 31 & 29 & 33 & 41 & 32 & 45 & 42 & 45 \\
\hline Loop & 26 & 21 & 29 & 26 & 32 & 38 & 31 & 42 & 36 & 42 \\
\hline Thiazide & 5 & 4 & 5 & 5 & 5 & 9 & 6 & 10 & $x x$ & $x x$ \\
\hline Alpha blocker & 7 & 7 & 7 & 4 & 5 & 10 & 10 & 10 & $x x$ & $x x$ \\
\hline Central alpha 2 agonist & 19 & 27 & 15 & 12 & 19 & 15 & 21 & 14 & $x x$ & $x x$ \\
\hline Hydralazine & 8 & 10 & 7 & 5 & 9 & 4 & 6 & 3 & $x x$ & $x x$ \\
\hline Minoxidil & 4 & 6 & 3 & 3 & 2 & 2 & 5 & 2 & $x x$ & $x x$ \\
\hline
\end{tabular}

Note: Values are percentages except for the $n$ row, which represents numbers of patients.

$\mathrm{ACEl}$, angiotensin converting enzyme inhibitor; $\mathrm{ARB}$, angiotensin receptor blocker; $C \mathrm{CB}$, calcium channel blocker; DHP, dihydropyridine; RAS, renin angiotensin system agent.

* Renin-inhibitors comprised $<1 \%$ of RAS agents from 2003-2008.

${ }^{\dagger}$ Potassium-sparing diuretics comprised $<1 \%$ of diuretic agents from 2003-2008.

$\mathrm{xx}$ represents less than 10 persons per cell; these percentages have been suppressed.

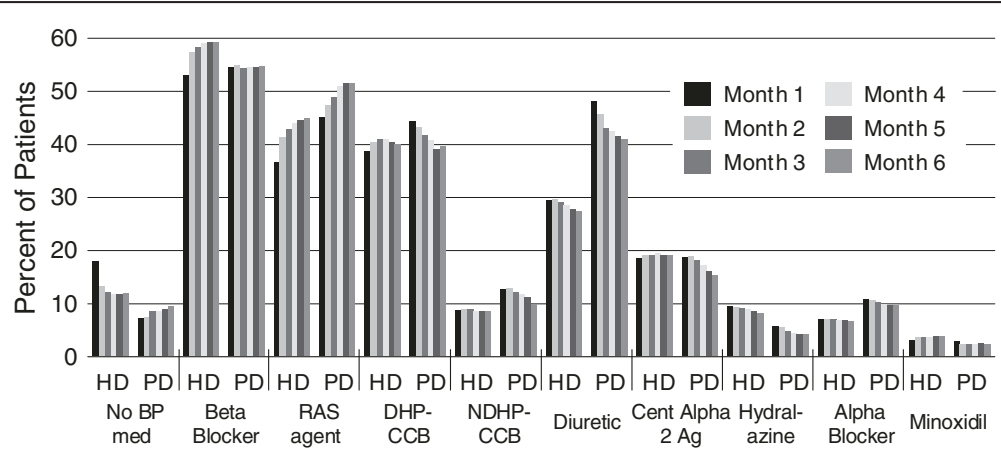

Figure 2 Percentages of hemodialysis and peritoneal dialysis patients prescribed blood pressure medication classes or specific agents over the first 6 months after dialysis initiation. BP, blood pressure; CCB, calcium channel blocker; Cent Alpha 2 Ag, central alpha 2 agonist; DHP, dihydropyridine; diuretic, any including thiazides, thiazide-like, loop, potassium-sparing; HD, hemodialysis; NDHP, non-dihydropyridine; PD, peritoneal dialysis; RAS, renin angiotensin system agent (angiotensin converting enzyme inhibitor or angiotensin receptor blocker). 
Prescription of central alpha 2 agonists and minoxidil decreased as age increased, but prescription of alpha blockers increased (data not shown).

Male and female hemodialysis patients were prescribed similar mean numbers of BP medications at month 6 (about 2.5), but female peritoneal dialysis patients received fewer prescriptions than male patients (2.4 vs. 2.7). Alpha blockers were prescribed more commonly for men ( $9 \%$ hemodialysis, $14 \%$ peritoneal dialysis) than for women ( $4 \%$ across modalities). Beta-blockers were prescribed more often for male than for female peritoneal dialysis patients ( $60 \%$ vs. 49 ; data not shown).

\section{Patterns by cardiovascular comorbidity and diabetes}

BP medication prescription varied by cardiovascular comorbidity and cardiovascular risk factors. Any betablocker, alpha-beta blockers, and loop diuretics were more commonly prescribed for patients with CHF, CVD, and diabetes across modalities than for patients without these conditions. RAS agents were more commonly prescribed for patients with than without diabetes across modalities, but prescriptions were similar for patients with and without CHF or CVD. DHP-CCBs were less frequently prescribed for patients with CHF and CVD than for patients without these conditions. Hydralazine was more frequently prescribed for hemodialysis and peritoneal dialysis patients with than without $\mathrm{CHF}$ (Table 3).

\section{Patterns in combination blood pressure medications}

Combination BP medication prescriptions were common. Beta-blockers were the only single medication class prescribed in $\geq 5 \%$ of patients. In the overall cohort, 43 BP medication class combinations were detected with classes defined as any beta-blocker, any RAS agent, DHP-CCBs, diuretics, central alpha 2 agonists, and remaining categories "other". Figure 3 shows the most common combinations prescribed for hemodialysis and

Table 3 Percentages of incident hemodialysis and peritoneal dialysis patients with prescriptions for specific blood pressure medications at 6 months by cardiovascular risk factor or disease, 2003-2008

\begin{tabular}{|c|c|c|c|c|c|c|c|c|c|c|c|c|c|c|}
\hline \multirow[b]{3}{*}{ Medication } & \multicolumn{7}{|c|}{ Hemodialysis, $n=12,159$} & \multicolumn{7}{|c|}{ Peritoneal dialysis, $n=913$} \\
\hline & \multirow[b]{2}{*}{ All } & \multicolumn{2}{|c|}{ CHF } & \multicolumn{2}{|c|}{ CVD } & \multicolumn{2}{|c|}{ Diabetes } & \multirow[b]{2}{*}{ All } & \multicolumn{2}{|c|}{$\mathrm{CHF}$} & \multicolumn{2}{|c|}{ CVD } & \multicolumn{2}{|c|}{ Diabetes } \\
\hline & & Yes & $\overline{\text { No }}$ & Yes & No & Yes & No & & Yes & $\overline{\text { No }}$ & Yes & No & Yes & No \\
\hline None & 12 & 10 & 13 & 11 & 13 & 10 & 15 & 10 & 7 & 10 & 7 & 11 & 5 & 14 \\
\hline \multicolumn{15}{|l|}{ Beta-blocker } \\
\hline Any & 59 & 65 & 55 & 64 & 53 & 62 & 54 & 55 & 69 & 51 & 67 & 48 & 61 & 48 \\
\hline Alpha-beta & 19 & 26 & 13 & 22 & 15 & 20 & 16 & 13 & 25 & 10 & 17 & 11 & 16 & 10 \\
\hline Beta not alpha & 41 & 39 & 42 & 43 & 38 & 42 & 38 & 42 & 44 & 41 & 51 & 37 & 45 & 38 \\
\hline \multicolumn{15}{|l|}{$\operatorname{RAS}^{*}$} \\
\hline Any & 45 & 45 & 45 & 44 & 46 & 49 & 39 & 52 & 54 & 51 & 55 & 50 & 57 & 46 \\
\hline ACEl & 32 & 32 & 31 & 31 & 32 & 34 & 27 & 33 & 36 & 32 & 34 & 32 & 35 & 30 \\
\hline$A R B$ & 17 & 16 & 17 & 16 & 18 & 19 & 14 & 23 & 22 & 23 & 25 & 22 & 26 & 19 \\
\hline \multicolumn{15}{|l|}{ CCB } \\
\hline Any & 49 & 44 & 52 & 45 & 52 & 49 & 47 & 50 & 43 & 51 & 48 & 51 & 54 & 44 \\
\hline $\mathrm{DHP}$ & 40 & 36 & 43 & 37 & 43 & 41 & 39 & 40 & 35 & 41 & 38 & 41 & 42 & 37 \\
\hline Non-DHP & 9 & 8 & 9 & 8 & 9 & 9 & 8 & 10 & 8 & 10 & 10 & 10 & 13 & 7 \\
\hline \multicolumn{15}{|l|}{ Diuretic $^{\dagger}$} \\
\hline Any & 28 & 31 & 24 & 28 & 26 & 31 & 21 & 41 & 55 & 37 & 52 & 35 & 50 & 32 \\
\hline Loop & 26 & 30 & 22 & 26 & 24 & 29 & 19 & 38 & 53 & 34 & 50 & 33 & 47 & 29 \\
\hline Thiazide & 5 & 5 & 4 & 4 & 5 & 5 & 3 & 9 & 13 & 8 & 13 & 6 & 12 & 5 \\
\hline Alpha blocker & 7 & 6 & 7 & 7 & 6 & 7 & 7 & 10 & 8 & 10 & 12 & 9 & 11 & 7 \\
\hline Central alpha 2 agonist & 19 & 18 & 20 & 17 & 21 & 20 & 18 & 15 & 14 & 16 & 14 & 16 & 16 & 14 \\
\hline Hydralazine & 8 & 11 & 6 & 9 & 7 & 9 & 7 & 4 & 9 & 3 & 5 & 4 & 6 & 3 \\
\hline Minoxidil & 4 & 3 & 4 & 3 & 5 & 4 & 5 & 2 & 2 & 3 & 1 & 3 & 2 & 2 \\
\hline
\end{tabular}

Note: Values are percentages except for the $n$ row, which represents number of individuals.

ACEl, angiotensin converting enzyme inhibitor; ARB, angiotensin receptor blocker; CCB, calcium channel blocker; DHP, dihydropyridine; RAS, renin angiotensin system agent.

Renin-inhibitors comprised $<1 \%$ of RAS agents from 2003-2008.

${ }^{\dagger}$ Potassium-sparing diuretics comprised $<1 \%$ of diuretic agents from 2003-2008. 


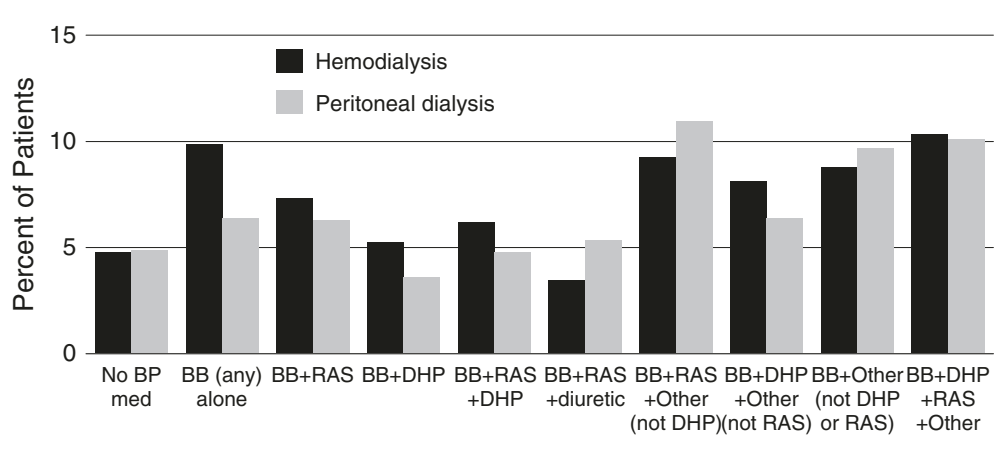

Figure 3 Blood pressure medications (single or in combination) prescribed for $\geq \mathbf{5} \%$ of hemodialysis or peritoneal dialysis patients with at least one blood pressure medication prescription. BB, beta blocker; BP, blood pressure; DHP, dihydropyridine; diuretic, any including thiazides, thiazide-like, loop, potassium-sparing; RAS, renin angiotensin system agent (angiotensin converting enzyme inhibitor or angiotensin receptor blocker).

peritoneal dialysis patients. As beta-blockers and RAS agents were overall the most frequently prescribed medications, when combinations were further collapsed to beta-blockers, RAS agents, and other, regimens including beta-blockers with and without RAS agents were the most common (Figure 4).

\section{Continuing blood pressure medications after 6 months}

We evaluated the percentage of patients who survived and continued the same BP medication at 12 and 24 months as at 6 months. Of all patients prescribed a beta-blocker at 6 months, $89 \%$ and $76 \%$ of those who survived at 12 and 24 months, respectively, continued beta-blocker prescriptions. In comparison, 79\% and 59\% continued DHP-CCB prescriptions and 83\% and 66\% RAS agent prescriptions (data not shown).

\section{Patterns for 2007-2008}

We evaluated the percentages of patients prescribed various BP medication classes and the most frequently prescribed agents in those classes at 6 months in the most current timeframe, 2007-2008, to evaluate trends over time. Compared with the overall timeframe of 2003-2008, prescription of beta-blockers across modalities and of ACEIs and loop diuretics in peritoneal dialysis patients were more frequent in 2007-2008. Percentages of patients prescribed ARBs, DHP-CCBs, alpha blockers, and central 2 alpha agonists remained stable (Table 4).

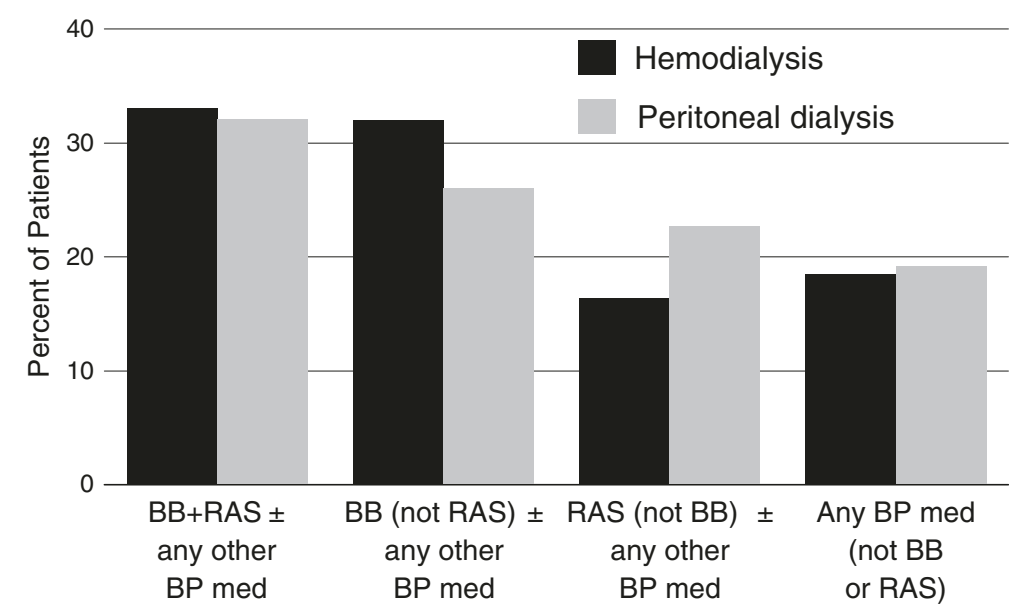

Figure 4 Blood pressure medication combinations prescribed for $\geq 10 \%$ of hemodialysis or peritoneal dialysis patients with at least one blood pressure medication prescription. BB, beta blocker; BP, blood pressure; RAS, renin angiotensin system agent (angiotensin converting enzyme inhibitor or angiotensin receptor blocker). 
Table 4 Percentages of hemodialysis and peritoneal dialysis patients prescribed specific blood pressure medications within frequently prescribed classes, ${ }^{*}$ 2007-2008

\begin{tabular}{|c|c|c|c|c|}
\hline \multirow[b]{2}{*}{ Class and medication } & \multicolumn{2}{|c|}{$\begin{array}{l}\text { Hemodialysis } \\
\text { patients }\end{array}$} & \multicolumn{2}{|c|}{$\begin{array}{c}\text { Peritoneal } \\
\text { dialysis patients }\end{array}$} \\
\hline & Class & Agent & Class & Agent \\
\hline Beta blockers & 65.6 & & 60.4 & \\
\hline Metoprolol succinate & & 25.5 & & 32.8 \\
\hline Metoprolol tartrate & & 27.7 & & 25.8 \\
\hline Carvedilol (alpha-beta blocker) & & 24.7 & & 22.7 \\
\hline Atenolol & & 11.0 & & 7.8 \\
\hline Labetalol (alpha-beta blocker) & & 9.1 & & 7.0 \\
\hline$\%$ of class represented & & 98.0 & & 96.1 \\
\hline ACEIS & 31.4 & & 39.6 & \\
\hline Lisinopril & & 66.6 & & 60.7 \\
\hline Enalapril & & 9.7 & & 1.2 \\
\hline Benazepril & & 9.5 & & 16.7 \\
\hline Ramipril & & 4.6 & & 7.1 \\
\hline Fosinopril & & 4.4 & & 4.8 \\
\hline Quinapril & & 4.3 & & 7.1 \\
\hline$\%$ of class represented & & 99.1 & & 97.6 \\
\hline ARBs & 17.0 & & 23.1 & \\
\hline Valsartan & & 40.4 & & 36.7 \\
\hline Losartan & & 31.4 & & 38.7 \\
\hline Irbesartan & & 11.0 & & 8.2 \\
\hline Olmesartan & & 9.2 & & 4.1 \\
\hline Telmisartan & & 5.3 & & 8.2 \\
\hline Candesartan & & 2.7 & & 4.1 \\
\hline$\%$ of class represented & & 100 & & 100 \\
\hline DHP-CCBs & 43.4 & & 39.2 & \\
\hline Amlodipine & & 66.2 & & 61.4 \\
\hline Nifedipine & & 23.2 & & 21.7 \\
\hline Felodipine & & 5.6 & & 8.4 \\
\hline Nisoldipine & & 4.0 & & 4.8 \\
\hline$\%$ of class represented & & 99.0 & & 96.3 \\
\hline Loop diuretics & 29.2 & & 45.3 & \\
\hline Furosemide & & 84.7 & & 89.6 \\
\hline Bumetanide & & 10.4 & & 6.3 \\
\hline Torsemide & & 4.9 & & 4.2 \\
\hline$\%$ of class represented & & 100 & & 100 \\
\hline Central alpha 2 agonist & 19.5 & & 12.3 & \\
\hline Clonidine & & 98.9 & & 96.2 \\
\hline Methyldopa & & 0.8 & & 3.8 \\
\hline$\%$ of class represented & & 99.7 & & 100 \\
\hline Alpha blockers & 6.6 & & 11.8 & \\
\hline Doxazosin & & 63.5 & & 68.0 \\
\hline Terazosin & & 33.8 & & 32.0 \\
\hline
\end{tabular}

Table 4 Percentages of hemodialysis and peritoneal dialysis patients prescribed specific blood pressure medications within frequently prescribed classes, ${ }^{*}$ 2007-2008 (Continued)

\begin{tabular}{|c|c|c|c|}
\hline Prazosin & & 2.7 & 0.0 \\
\hline$\%$ of class represented & & 100 & 100 \\
\hline Hydralazine & 11.1 & & \\
\hline$\%$ of class represented & & 100 & 100 \\
\hline \multicolumn{4}{|c|}{$\begin{array}{l}\text { Note: Values are percentages. } \\
\text { ACEI, angiotensin converting enzyme inhibitor; ARB, angiotensin receptor } \\
\text { blocker; DHP-CCB, dihydropyridine calcium channel blocker. } \\
\text { "Prescribed in at least } 10 \% \text { of hemodialysis or peritoneal dialysis patients at } \\
\text { month } 6 \text { after dialysis initiation. }\end{array}$} \\
\hline
\end{tabular}

The most frequently prescribed beta-blockers in 2007-2008 were metoprolol succinate (sustained release), metoprolol tartrate (non-sustained release), and the alpha-beta blocker carvedilol. Lisinopril was the predominant ACEI prescribed, and losartan and valsartan the predominant ARBs. Amlodipine was the predominant DHP-CCB prescribed and doxazosin the predominant alpha blocker. Furosemide was virtually the only loop diuretic and clonidine the only central alpha 2 agonist prescribed.

\section{Discussion}

Our study describes BP medication use in the first 6 months after dialysis initiation in a large population of hemodialysis and a smaller population of peritoneal dialysis patients. In hemodialysis patients, beta-blocker prescriptions increased over 6 months, DHP-CCB prescriptions remained stable, and diuretic and hydralazine prescriptions decreased slightly. In peritoneal dialysis patients, the percentage prescribed beta-blockers remained stable over 6 months and prescriptions of diuretics, DHP-CCBs, and central alpha 2 agonists steadily decreased. Prescription of RAS agents increased in both cohorts. The mean number of prescribed medications increased for hemodialysis but decreased for peritoneal dialysis patients over 6 months. Although prescription patterns appeared to stabilize in both cohorts by months 5 and 6, a substantial percentage of patients prescribed specific medications at month 6 were no longer prescribed those medications at month 24. Combination prescriptions were common in both cohorts. We found trends toward increased beta-blocker use in both cohorts and ACEI and loop diuretic use in peritoneal dialysis patients comparing 2003-2008 data with 2007-2008 data.

Our study has several strengths. First, data were obtained from the third-largest dialysis provider in the US, allowing us to evaluate differences in BP medication prescriptions between hemodialysis and peritoneal dialysis patients and to stratify our analyses by several characteristics, including baseline CHF, CVD, and diabetes, which 
influence prescription of cardioprotective medications [14]. Second, our analysis provides in-depth examination of contemporary prescribing patterns, which is important because BP medication prescription is influenced by available evidence on efficacy and by generic availability. Third, we evaluated use of combination prescriptions, which was not reported in previous studies.

Our results reflect a purely descriptive analysis; results have not been adjusted for important patient characteristics. In addition, our study did not identify reasons for apparent differences in prescription patterns by modality over the first 6 months. Some of the apparent differences may stem from differing baseline characteristics. For instance, beta-blockers were prescribed for more hemodialysis than peritoneal dialysis patients. Hemodialysis patients were older, on average, and beta-blockers were more likely to be prescribed for older patients. Hemodialysis patients had more CHF, CVD, and diabetes, and beta-blockers were more likely to be prescribed in patients with these conditions.

We hypothesized that the mean number of medications would decrease over time as volume control improved. However, the mean number of medications increased for hemodialysis patients but decreased for peritoneal dialysis patients over the first 6 months. Residual renal function declines more rapidly with hemodialysis than with peritoneal dialysis $[18,19]$, possibly necessitating additional BP medications in hemodialysis patients over the first 6 months. For peritoneal dialysis patients, only prescription of RAS agents increased, and prescription of all other medications declined or remained stable (beta-blockers). Perhaps maintained residual renal function with RAS agents, along with better volume control, allowed for reduced medications in peritoneal dialysis patients.

Our findings regarding BP medication patterns among dialysis patients with CHF are consistent with Wetmore et al., who found that CHF was associated with a 9\% increase in odds of beta-blocker use and no increase in RAS agent use in dually eligible (Medicare/Medicaid) prevalent hypertensive dialysis patients [14]. Our stratified analysis also showed much higher use of any betablocker in hemodialysis and peritoneal dialysis patients with than without $\mathrm{CHF}$, driven mainly by prescription of beta-blockers with alpha activity. Similarly, we found little difference in RAS agent use among patients with or without CHF. Wetmore et al. also showed higher odds of RAS agent use in prevalent dialysis patients with than without diabetes [14], consistent with results from our stratified analysis.

In month $1,48 \%$ of peritoneal dialysis and $29 \%$ of hemodialysis patients received diuretics, despite the same estimated glomerular filtration rate at dialysis initiation. However, diuretic use declined more rapidly over 6 months in peritoneal dialysis patients, which was unexpected since decline in renal function is slower in these patients [19]. Loop diuretics have not been shown to preserve residual renal function [20], and an interesting question relates to whether increased prescription of RAS agents in peritoneal dialysis patients after dialysis initiation led to decreased glomerular filtration rate or improved BP enough to obviate need for diuretics in some patients. One analysis of Dialysis Outcomes and Practice Patterns Study (DOPPS) data showed that diuretic use increased in incident US hemodialysis patients 1997-2001 (23\% to 30\%), and use was associated with lower interdialytic weight gain and lower odds of hyperkalemia [21]. Similarly, our 2007-2008 data showed diuretic prescriptions for $29 \%$ of DCI hemodialysis patients at month 6.

Comparing BP medication prescription data in our incident cohort of DCI dialysis patients in 2007-2008 with a large prevalent cohort of US dialysis patients enrolled in Medicare Part D in 2007 ( $n=158,702)$, we found similar proportions prescribed beta-blockers (DCI: hemodialysis, 66\%; peritoneal dialysis, $60 \%$ vs. USRDS: $64 \%$ ), ARBs (DCI: hemodialysis, 17\%; peritoneal dialysis, $23 \%$ vs. USRDS: 21\%), and DHP-CCBs (DCI: hemodialysis, 43\%; peritoneal dialysis, 39\% vs. USRDS: 46\%), but much higher proportions prescribed diuretics (DCI: hemodialysis, 29\%; peritoneal dialysis, $45 \%$ loop diuretics vs. USRDS: $17 \%$ any diuretic), and lower proportions prescribed ACEIs (DCI: hemodialysis, 31\%; peritoneal dialysis $40 \%$ vs. USRDS: $38 \%$ ) and central alpha 2 agonists (DCI: hemodialysis, $20 \%$; peritoneal dialysis, $12 \%$ vs. USRDS: $23 \%$ ) [15]. The higher proportions prescribed diuretics in our cohorts presumably reflect higher residual renal function in the DCI incident than in the USRDS prevalent population.

Several observational studies using incident cohorts from Dialysis Morbidity and Mortality Wave 2 data have evaluated the influence of single BP or cardioprotective medications on long-term outcomes with exposure to a BP medication at a single point [6,8-10]; based on our findings, results may be confounded by substantial changes in BP medication prescription patterns over the first months of dialysis. We also found that a substantial percentage of patients prescribed specific medications at 6 months were no longer prescribed those medications at 24 months. This clearly requires further study. However, observational studies of BP medications in prevalent patients using a single exposure period and long follow-up periods may also be confounded [11].

Our findings regarding distributions of use for specific agents differed from those of a prior study of prevalent dialysis patients [14]. Regarding beta-blockers, we found higher use of metoprolol products and carvediol and less of atenolol and labetalol. As metoprolol succinate (sustained release) is now available in a generic version, one would expect prescription patterns to shift toward higher use if this study were repeated in a more contemporary 
timeframe. Carvedilol, a beta-blocker with alpha activity, reduces left ventricular volume in dialysis patients with dilated cardiomyopathy [22]. Generic carvediol became widely available in 2007 , partially explaining its higher use in our cohort.

Prescription of multiple BP medications was common across modalities. Prescription patterns varied for each agent and by modality in the first 6 months after dialysis initiation. These patterns are important to consider in the design of comparative-effectiveness studies of BP medications in dialysis patients. As prescription patterns differ distinctly, hemodialysis and peritoneal dialysis patients should not be grouped together in studies evaluating the effect of medications on outcomes. On a population level, prescription patterns varied by medication class in the first 6 months, but appeared to stabilize by months 5 and 6 . This suggests that a time-dependent approach should be used in assessing medication effect in incident patients if follow-up starts at dialysis initiation. Our evaluation of changes over time in individual patients prescribed betablockers, RAS agents, and/or DHP-CCBs at month 6 showed that time-dependent changes in prescriptions should be considered even after 6 months, particularly if follow-up time is longer than 6-12 months. Impact of and possible interactions with other concurrent medications should also be considered in designing future studies.

Our study has some limitations. Although characteristics of our cohort were similar to national characteristics [1], DCI medication prescribing patterns may not reflect patterns in other dialysis facilities. Since data were derived from EMRs, we know only that medications were prescribed, not whether patients consumed them. Further, accuracy of DCI outpatient medication lists may vary by dialysis unit and may not correctly reflect recent BP medication prescription changes made by nondialysis health care providers. In addition, we required patients to survive for 6 months in order to evaluate BP management in a stable cohort; in analyses of BP medications at 12 and 24 months, we required patients to survive to those points. Prescription patterns may differ for surviving patients and for patients who died. The number of included peritoneal dialysis patients was relatively small, which limited interpretation of results in some race and ethnicity groups. Finally, although we stratified our findings by important patient characteristics, our analyses are largely descriptive and do not adjust for multiple characteristics that could influence medication prescription. Thus, the data from hemodialysis and peritoneal dialysis patients are not directly comparable, because the characteristics of these populations differ.

\section{Conclusions}

In conclusion, our study evaluates BP medication prescription patterns in hemodialysis and peritoneal dialysis patients in the first 6 months after dialysis initiation. Observational studies attempting to compare medication effectiveness should account for the time-varying nature of BP medication prescriptions, particularly in the first 6 months and if follow-up extends beyond 6-12 months. Given that most patients are prescribed multiple medications, investigators should consider study designs that incorporate medication combinations. Finally, prescription patterns after dialysis initiation differ by modality, and study designs should account for these differences. Further research is necessary to determine the effects of BP medication patterns on intermediate outcomes such as BP variability, and key clinical outcomes such as cardiovascular mortality and hospitalization, sudden cardiac death, and quality of life.

\section{Competing interests}

The authors report no conflicts of interest with the study subject matter.

\section{Authors' contributions}

All authors participated in study conception or design, or analysis and interpretation of data, or both; Dr. St. Peter drafted the manuscript and the other authors provided intellectual content of critical importance to the work. All authors approved the version to be submitted.

\section{Acknowledgments}

The Developing Evidence to Inform Decisions about Effectiveness (DECIDE) Network Patient Outcomes in ESRD Study was supported by the Agency for Healthcare Research and Quality (AHRQ) contract HHSA290200500341I, Task Order 6.

Dr. Shafi was supported by grant K23-DK-083514 from the National Institute for Diabetes, Digestive and Kidney Diseases (NIDDK), Bethesda MD. Dr. Michels was supported by a Postdoctoral Full Fellowship Abroad Grant (KFB 11.005) of the Dutch Kidney Foundation (Nierstichting), Amerstdam, The Netherlands.

AHRQ: Identifiable information, on which this report, presentation, or other form of disclosure is based, is confidential and protected by federal law, Section 903(c) of the Public Health Service Act, 42 USC 299a-1(c). Any identifiable information that is knowingly disclosed is disclosed solely for the purpose for which it has been supplied. No identifiable information about any individual supplying the information or described in it will be knowingly disclosed except with the prior consent of that individual.

USRDS: The data reported here have been supplied by the United States Renal Data System (USRDS). The interpretation and reporting of these data are the responsibility of the author(s) and in no way should be seen as an official policy or interpretation of the U.S. government.

The DEcIDE Network Patient Outcomes in End-Stage Renal Disease Study Team consists of members from the Johns Hopkins University, Baltimore, Maryland (L. Ebony Boulware, Karen Bandeen-Roche, Courtney Cook, Josef Coresh, Deidra Crews, Patti Ephraim, Bernard Jaar, Jeonyong Kim, Yang Liu, Jason Luly, Aidan McDermott, Paul Scheel, Tariq Shafi, Stephen M. Sozio, Albert Wu, Jing Zhou); University of California, San Francisco, California (Neil Powe); Chronic Disease Research Group, Minneapolis, Minnesota (Allan Collins, Robert Foley, David Gilbertson, Haifeng Guo, Charles Herzog, Jiannong Liu, Wendy St. Peter); Cleveland Clinic Foundation, Cleveland, Ohio (Joseph Nally, Susana Arrigain, Stacey Jolly, Vicky Konig, Xiaobo Liu, Sankar Navaneethan, Jesse Schold,); University of New Mexico (Philip Zager); Tufts University, Boston, Massachusetts (Dana Miskulin, Klemens Meyer); University of Miami, Miami, Florida (Julia Scialla); University of Manitoba, Winnipeg, Manitoba (Navdeep Tangri); and Academic Medical Center, Amsterdam, The Netherlands (Wieneke Michels).

Parts of this work were presented at the 2012 Annual Meeting of the American Society of Nephrology in San Diego, California, November 1 through 4, 2012. The authors thank Chronic Disease Research Group colleagues Pamela Giles for manuscript preparation and Nan Booth, MSW, $\mathrm{MPH}, \mathrm{ELS}$, for manuscript editing. The authors express their gratitude to the staff and patients of Dialysis Clinic Inc. 


\section{Author details}

'University of Minnesota College of Pharmacy, Minneapolis, MN, USA.

${ }^{2}$ Chronic Disease Research Group, Minneapolis Medical Research Foundation, 914 South 8th Street, Suite S4.100, Minneapolis, MN 55404, USA. ${ }^{3}$ Division of Nephrology, Johns Hopkins University School of Medicine, Baltimore, MD, USA. ${ }^{4}$ Welch Center for Prevention, Epidemiology, and Clinical Research, Johns Hopkins Medical Institutions, Baltimore, MD, USA. ${ }^{5}$ Department of Epidemiology, Johns Hopkins Bloomberg School of Public Health, Baltimore, MD, USA. ${ }^{6}$ Division of General Internal Medicine, Johns University Hopkins School of Medicine, Baltimore, MD, USA. ${ }^{7}$ Department of Biostatistics, Johns Hopkins Bloomberg School of Public Health, Baltimore, MD, USA. ${ }^{8}$ Division of Nephrology, Tufts University School of Medicine, Boston, MA, USA. ${ }^{9}$ Department of Medicine, University of Miami Miller School of Medicine, Miami, FL, USA. ${ }^{10}$ Department of Medicine, Division of Nephrology, Seven Oaks General Hospital, University of Manitoba, Winnipeg, Manitoba, Canada. ${ }^{11}$ Nephrology Center of Maryland, Baltimore, MD, USA. ${ }^{12}$ Division of Nephrology, Department of Medicine, Academic Medical Center, Amsterdam, The Netherlands. ${ }^{13}$ Department of Health Policy and Management, Johns Hopkins Bloomberg School of Public Health, Baltimore, MD, USA.

${ }^{14}$ Department of International Health, Johns Hopkins Bloomberg School of Public Health, Baltimore, MD, USA. ${ }^{15}$ Department of Surgery, Johns Hopkins University School of Medicine, Baltimore, MD, USA.

Received: 8 July 2013 Accepted: 4 November 2013

Published: 12 November 2013

\section{References}

1. U.S. Renal Data System: USRDS 2012 Annual Data Report: Atlas of Chronic Kidney Disease \& End-Stage Renal Disease in the United States. 2012th edition. Bethesda, MD: National Institutes of Health, National Institute of Diabetes and Digestive and Kidney Diseases; 2012.

2. Agarwal R, Sinha AD: Cardiovascular protection with antihypertensive drugs in dialysis patients: systematic review and meta-analysis. Hypertension 2009, 53:860-866.

3. Heerspink HJ, Ninomiya T, Zoungas S, et al: Effect of lowering blood pressure on cardiovascular events and mortality in patients on dialysis: a systematic review and meta-analysis of randomised controlled trials. Lancet 2009, 373:1009-1015.

4. Iseki K, Shoji T, Nakai S, Watanabe Y, Akiba T, Tsubakihara Y: Higher survival rates of chronic hemodialysis patients on anti-hypertensive drugs. Nephron Clin Pract 2009, 113:c183-c190.

5. Nakao K, Makino H, Morita S, et al: Beta-blocker prescription and outcomes in hemodialysis patients from the Japan Dialysis Outcomes and Practice Patterns Study. Nephron Clin Pract 2009, 113:c132-c139.

6. Abbott KC, Trespalacios FC, Agodoa LY, Taylor AJ, Bakris GL: beta-Blocker use in long-term dialysis patients: association with hospitalized heart failure and mortality. Arch Intern Med 2004, 164:2465-2471.

7. Lopes AA, Bragg-Gresham JL, Ramirez SP, et al: Prescription of antihypertensive agents to haemodialysis patients: time trends and associations with patient characteristics, country and survival in the DOPPS. Nephrol Dial Transplant 2009, 24:2809-2816.

8. Griffith TF, Chua BS, Allen AS, Klassen PS, Reddan DN, Szczech LA: Characteristics of treated hypertension in incident hemodialysis and peritoneal dialysis patients. Am J Kidney Dis 2003, 42:1260-1269.

9. Ishani A, Herzog CA, Collins AJ, Foley RN: Cardiac medications and their association with cardiovascular events in incident dialysis patients: cause or effect? Kidney Int 2004, 65:1017-1025.

10. Kestenbaum B, Gillen DL, Sherrard DJ, Seliger S, Ball A, Stehman-Breen C: Calcium channel blocker use and mortality among patients with end-stage renal disease. Kidney Int 2002, 61:2157-2164.

11. Foley RN, Herzog CA, Collins AJ: Blood pressure and long-term mortality in United States hemodialysis patients: USRDS Waves 3 and 4 Study. Kidney Int 2002, 62:1784-1790.

12. Manley HJ, Garvin CG, Drayer DK, et al: Medication prescribing patterns in ambulatory haemodialysis patients: comparisons of USRDS to a large not-for-profit dialysis provider. Nephrol Dial Transplant 2004, 19:1842-1848.

13. Rahman M, Griffin V: Patterns of antihypertensive medication use in hemodialysis patients. Am J Health Syst Pharm 2004, 61:1473-1478.

14. Wetmore JB, Mahnken JD, Mukhopadhyay P, et al: Geographic variation in cardioprotective antihypertensive medication usage in dialysis patients. Am J Kidney Dis 2011, 58:73-83.
15. Frankenfield DL, Weinhandl ED, Powers CA, Howell BL, Herzog CA, St.Peter WL: Utilization and costs of cardiovascular disease medications in dialysis patients in Medicare Part D. Am J Kidney Dis 2012, 59:670-681.

16. Sood MM, Battistella M, Lok CE: Patterns of cardioprotective medication prescription in incident hemodialysis patients. Int Urol Nephrol 2009, 41:1021-1027.

17. Boulware EL, Tangri N, Ephraim PL, et al: Comparative effectiveness studies to improve clinical outcomes in end stage renal disease: the DECIDE patient outcomes in end stage renal disease study. BMC Nephrol 2012, 13:167.

18. Thomas J, Teitelbaum I: Preservation of residual renal function in dialysis patients. Adv Perit Dial 2011, 27:112-117.

19. Jansen MA, Hart AA, Korevaar JC, Dekker FW, Boeschoten EW, Krediet RT Predictors of the rate of decline of residual renal function in incident dialysis patients. Kidney Int 2002, 62:1046-1053.

20. Medcalf JF, Harris KP, Walls J: Role of diuretics in the preservation of residual renal function in patients on continuous ambulatory peritoneal dialysis. Kidney Int 2001, 59:1128-1133.

21. Bragg-Gresham JL, Fissell RB, Mason NA, et al: Diuretic use, residual renal function, and mortality among hemodialysis patients in the Dialysis Outcomes and Practice Pattern Study (DOPPS). Am J Kidney Dis 2007, 49:426-431.

22. Cice G, Ferrara L, Di Benedetto A, et al: Dilated cardiomyopathy in dialysis patients-beneficial effects of carvedilol: a double-blind, placebo-controlled trial. J Am Coll Cardiol 2001, 37:407-411.

doi:10.1186/1471-2369-14-249

Cite this article as: St. Peter et al:: Patterns in blood pressure medication use in US incident dialysis patients over the first 6 months. BMC Nephrology 2013 14:249.

\section{Submit your next manuscript to BioMed Central and take full advantage of:}

- Convenient online submission

- Thorough peer review

- No space constraints or color figure charges

- Immediate publication on acceptance

- Inclusion in PubMed, CAS, Scopus and Google Scholar

- Research which is freely available for redistribution 